\title{
The changing face of Dentistry in the COVID-19 pandemic
}

SADJ November 2020, Vol. 75 No. 10 p530

NH Wood

Managing Editor of the SADJ

The year is drawing to a close and with it we present to you the final issue of the South African Dental Journal for 2020. This year saw us face the terrible COVID-19 pandemic. As oral healthcare practitioners we have been challenged in many ways, and this placed many of us under extreme pressure. Unfortunately we are just starting to see a new rise, or second wave, in the numbers of positive COVID-19 cases being reported. This means that the pressure is not about to be released, and we continue to navigate these uncharted waters into 2021.

In February we were uncertain about the impact of COVID-19 on our healthcare systems and our country, however we have now seen the course and the progression of this disease in many countries, and we know the reach it has and the devastating infection it causes. In light of this I am reminding you of the required infection control processes and procedures that need to be in place to protect yourself, co-workers and your patients.

The same is true for the various dental schools throughout the country: The staff and students within these schools have faced unprecedented challenges to break through the COVID-19 obstruction of academic activity, to be able to continue with the high-quality training of students in different disciplines of oral healthcare. All teaching has moved to online platforms and clinical competency training in contact sessions are strictly controlled and monitored according to policies and procedures specifically developed for the COVID-19 pandemic. This colossal effort must be recognised because many have made, and are still making, personal sacrifices in an immense effort to see to it that a continuous stream of oral healthcare graduates is maintained.

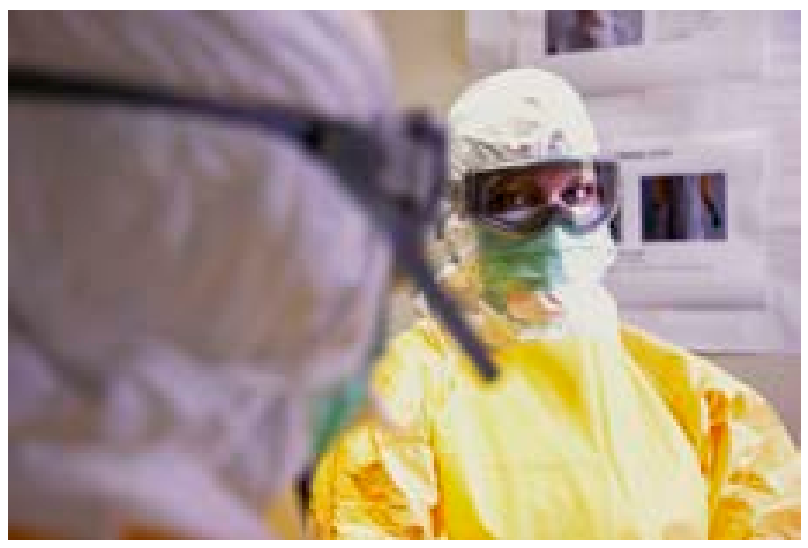

Neil H Wood: Managing editor. Email: neil.wood@smu.ac.za
However, I must urge these institutions of higher learning to look after their staff and to take care of the wellbeing of these individuals, both physically and mentally. It is because of the enormous efforts and the unrelenting pressure to produce deliverables and outcomes based on pre-COVID-19 conventions and designs, that levels of exhaustion may be precariously high; and our clinicians and students must be protected against burnout. Protocols and measures must be put in place by these institutions to guard against these unintended deleterious outcomes.

There is no doubt in my mind that COVID-19 has radically changed the way clinical dentistry, academic dentistry and clinical teaching and training is conducted, and that there will be no return to the ways of the past.

The South African Dental Journal's new online platform is now fully operational and functioning. Please take a moment to visit the new online platform on which the South African Dental Journal is hosted at https:// journals.assaf.org.za/index.php/sadj/index and register as a reviewer or author. I would also appreciate any feedback that you as a reader, author or reviewer may have on this new platform, as we are always looking for ways to improve on the SADJ delivery. This issue of the SADJ contains another healthy mix of content for our readers along with provision for acquiring CPD points.

Our regular contributors once more support us with interesting material that includes the radiology case prepared by Prof $\mathrm{C}$ Nortjé, and some views on recently published material compiled by Prof Yengopal.

A sincere word of thanks to all our authors, reviewers and other contributors for the participation in making the 2020 edition of the SADJ a success. We truly have a journal that belongs to our dental community. I would like to express my gratitude for the hard work and dedication put into the 2020 edition of the SADJ by all the members in the team, Dr Nthabiseng Metsing, Mr Dumi Ngoepe, Mrs Ann Bayman, as well as our colleagues at the production office Ms Reine Combrinck and Mr Rene Smulders. Thanks also to Prof Bill Evans for his contributions toward the cover-page and his gracious guidance. The year saw many changes and improvements to our journal, and may it long continue.

From the Editorial office we would like to wish our readers and our members a safe and peaceful festive season. We look forward to the new year to achieve and celebrate new success and development with you. 